\title{
Interactions géniteur $\times$ population des partenaires II. Détection par des expériences de sélection
}

\author{
J.M. BRUN \\ I.N.R.A., Station d'Amélioration génétique des Animaux, \\ B.P. 12, F 31320 Castanet-Tolosan
}

\begin{abstract}
Résumé
On examine l'intérêt des expériences de sélection pour l'étude des interactions entre les géniteurs d'une population $\mathrm{P}_{\mathrm{a}}$ et la population de leurs partenaires $\left(\mathrm{P}_{\mathrm{a}}\right.$ ou $\left.\mathrm{P}_{\mathrm{b}}\right)$. La première partie consiste à établir un modèle d'expérience pour calculer les paramètres réalisés de cette interaction : variances et covariance des aptitudes générales à la combinaison (A.G.C.) d'un géniteur en pur et en croisement. Dans la deuxième partie, on recherche des méthodes d'estimation de ces paramètres à partir des expériences disponibles dans la littérature, en tenant compte de leurs écarts au modèle d'expérience proposé (expériences de sélection intra-population conduites sur 2 populations utilisées en croisement et expériences de sélection récurrente et réciproque). On en déduit un indicateur d'interaction facile à calculer, car uniquement fonction de la réponse moyenne des populations parentales et de celle de leurs croisements. Il estime le coefficient de régression réalisé de l'A.G.C. en croisement sur l'A.G.C. intra-population (ou inversement). Cet indicateur contribuera à une analyse bibliographique du déterminisme de ces interactions à partir de résultats expérimentaux portant sur les animaux.
\end{abstract}

Mots clés : Interactions génotype $\times$ milieu, sélection, croisement.

\section{Summary \\ Interactions between breeding animals and populations of their mates. II. Estimation from selection experiments}

It is shown how selection experiments can be used to study the interactions between breeding animals from a given population $\left(P_{a}\right)$ and their potential mates in the same $\left(P_{a}\right)$ or another $\left(P_{b}\right)$ population. First, an experimental design is proposed for estimating the parameters of these interactions : the variances and covariance of the general combining ability (G.C.A.) of a sire in pure and crossbreeding. Secondly, methods of estimation of these parameters from selection experiments available in the literature are proposed. These experiments are the within population selection of 2 populations used in crossbreeding and the reciprocal recurrent selection. An index of interaction is proposed which is simply a function of the mean responses of the parental populations and of their crosses : this index estimates the regression coefficient of the G.C.A. in crossbreeding on the G.C.A. in purebreeding (or the reverse) and provides a useful tool for the future synthesis of data from the literature.

Key words : Genotype $\times$ environment interactions, selection, crossbreeding. 


\section{Introduction}

Appliquant le concept d'interaction génotype $\times$ milieu au cas où le terme de «milieu» désigne un milieu génétique, on postule l'existence d'interactions entre le génotype d'un géniteur et le type génétique de ses partenaires, c'est-à-dire sa population partenaire.

Ces interactions peuvent s'exprimer de façon statique, à une génération donnée : elles consistent en une différence de classement sur descendance d'un ensemble de géniteurs et en une différence de variabilité entre les descendances de ces géniteurs, selon la population partenaire. BRUN (1982) propose un modèle de cette interaction dans le cas où des mâles (par exemple) appartenant à une population $\mathrm{P}_{\mathrm{a}}$ se reproduisent en panmixie avec des femelles de 2 populations, la population $P_{a}$ elle-même et une autre population, $P_{b}$. Les paramètres de l'interaction géniteur de $P_{a} \times$ population partenaire $\left(\mathrm{P}_{\mathrm{a}}\right.$ ou $\left.\mathrm{P}_{\mathrm{b}}\right)$ sont les variances et covariance des aptitudes générales à la combinaison (A.G.C.) des géniteurs de $P_{a}$, intra-population $\left(\Gamma_{a a}\right)$ et en croisement avec $\mathrm{P}_{\mathrm{b}}\left(\Gamma_{\mathrm{ab}}\right): \mathrm{v}\left(\Gamma_{\mathrm{aa}}\right), \mathrm{v}\left(\Gamma_{\mathrm{ab}}\right)$ et $\operatorname{cov}\left(\Gamma_{\mathrm{aa}}, \Gamma_{\mathrm{ab}}\right)$.

Ces interactions s'expriment également, mais de façon dynamique, lors d'expériences de sélection, où les géniteurs d'une population sont sélectionnés dans un milieu génétique donné et se reproduisent dans un autre milieu génétique. C'est le cas, par exemple, de populations dans lesquelles la sélection des candidats se base sur des tests intra-population et qui sont ensuite utilisées en croisement ou inversement. On conçoit alors que le recours à des expériences de sélection puisse renseigner sur l'existence de ces interactions et permettre d'en évaluer l'importance. Le tableau 1 illustre l'application de cette méthode dans le cas de 2 milieux non génétiques pour calculer la corrélation génétique entre expressions du même caractère dans ces 2 milieux, paramètre de l'interaction génotype $\times$ milieu. Elle consiste à sélectionner pour le caractère dans les 2 milieux et à confronter les réponses directes, obtenues dans le milieu de sélection et les réponses corrélées obtenues dans l'autre milieu.

\section{TABleau 1}

Calcul de la corrélation génétique réalisée ( $\left.r \hat{g}_{12}\right)$ entre expressions d'un caractère dans 2 milieux (1 et 2) à partir d'expériences de sélection massale.

Calculation of the realised genetic correlation $\left(r \hat{g}_{12}\right)$ between the expressions of the same trait in 2 different environments ( 1 and 2) with mass selection.

\begin{tabular}{|c|c|c|c|}
\cline { 3 - 4 } \multicolumn{2}{c|}{} & \multicolumn{2}{c|}{ Milieu de mesure de la réponse } \\
\cline { 3 - 4 } \multicolumn{1}{c|}{} & 1 & 2 \\
\hline \multirow{3}{*}{$\begin{array}{c}\text { Milieu } \\
\text { de } \\
\text { délection }\end{array}$} & 1 & $\begin{array}{c}\text { Directe } \\
\mathbf{R}_{11}=\mathrm{i}_{1} \cdot \mathrm{h}_{1}^{2}\end{array}$ & $\begin{array}{c}\text { Corrélée } \\
\mathrm{R}_{21}=\mathrm{i}_{1} \cdot \mathrm{h}_{1} \cdot \mathrm{h}_{2} \cdot \mathrm{rg}_{12}\end{array}$ \\
\cline { 2 - 4 } & 2 & $\begin{array}{c}\text { Corrélée } \\
\mathrm{R}_{12} \cdot \mathrm{h}_{2} \cdot \mathrm{h}_{1} \cdot \mathrm{rg}_{12}\end{array}$ & $\begin{array}{c}\text { Directe } \\
\mathbf{R}_{22}=\mathrm{i}_{2} \cdot \mathrm{h}_{2}^{2}\end{array}$ \\
\hline
\end{tabular}

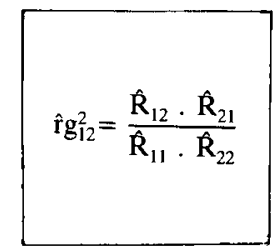

$R_{i j}$ est l'espérance de la réponse du caractère dans le milieu i à la sélection faite dans le milieu $j$, $\hat{R}_{i j}$ est la réponse réalisée.

$h_{j}^{2}$ et $i_{j}$ sont respectivement l'héritabilité du caractère (exprimé en unités d'écart-type) et l'intensité de la sélection dans le milieu $\mathrm{j}$.

$\mathrm{rg}_{12}$ est la corrélation génétique entre les expressions du caractère dans les milieux 1 et 2 . 
L'objet de cette publication est de montrer l'utilisation possible des résultats d'expériences de sélection pour détecter les interactions géniteur $\times$ population partenaire. Dans un premier temps, on propose un modèle d'expérience de sélection pour détecter ces interactions. Dans une deuxième temps, on recherche une méthode d'interprétation des résultats expérimentaux disponibles dans la littérature, en termes d'interactions géniteur $\times$ population partenaire : ces expériences ne réalisent généralement pas les conditions idéales du modèle proposé et on en discute les conséquences pour le test de l'interaction.

\section{Modèle d'expérience de sélection pour détecter l'interaction géniteur $\times$ population partenaire dans le cas de 2 populations}

\section{A. Protocole de l'expérience}

Soient 2 populations $\mathrm{P}_{\mathrm{a}}$ et $\mathrm{P}_{\mathrm{b}}$, d'individus diploïdes, supposées infiniment grandes et panmictiques, et un caractère quantitatif à déterminisme autosomal et non soumis à des effets indirects tels que les effets maternels. Soit $P_{a b}$ la population résultant du croisement entre $P_{a}$ et $P_{b}$. Considérons les interactions entre les géniteurs de $P_{a}$ et leur population partenaire, pouvant être $P_{a}$ ou $P_{b}$. Conformément aux principes du tableau 1 , sélectionnons 2 populations telles que $P_{a}$, l'une sur la base de tests (des candidats) intra-population et l'autre, de tests en croisement avec $P_{b}$ et observons dans chaque cas les réponses des populations $P_{a}$ et $P_{a b}, P_{b}$ n'étant pas elle-même sélectionnée.

La figure 1 (d'après GallaIs, 1978) décrit un cycle de sélection de $P_{a}$ à la génération $\mathrm{n}\left(\mathrm{P}_{\mathrm{a}}^{\mathrm{n}}\right)$, selon un système de test quelconque $\mathscr{T}_{i}$. Il se compose d'une phase de test des candidats à la sélection (phase I) suivie de sélection par troncature et d'une phase de reproduction panmictique intra-population pour former $P_{a}^{n+1}$ (phase II). Ce cycle s'accompagne d'une phase de croisement entre $\mathrm{P}_{\mathrm{a}}^{n+1}$ et $\mathrm{P}_{\mathrm{b}}^{+1}$ pour former $\mathrm{P}_{\mathrm{ab}}^{\mathrm{n}+1}$ (phase III) et ainsi réaliser le progrès génétique au niveau de la population $P_{a b}$. Notons $\mathcal{M}_{\mathrm{a} a}$ le système de reproduction permettant de passer de $\mathrm{P}_{\mathrm{a}}^{\mathrm{a}}$ a $\mathrm{P}_{\mathrm{ab}}^{\mathrm{n}}{ }^{+1}$ (c'est la phase II) et $\mu_{a b}$ celui qui permet de passer de $P_{a}^{n}$ à $P_{a b}^{n+1}$ (c'est la succession des deux phases II et III).

On envisagera 2 types de test intra-population (un test $\mathscr{T}_{\mathrm{a}}$, sur valeur phénotypique individuelle et un test $\mathscr{T}_{\text {aa }}$ sur A.G.C., où l'on mesure la valeur phénotypique moyenne de la descendance d'un candidat de $P_{a}$ avec un échantillon de femelles de $P_{a}$ ) et un seul type de test en croisement, sur A.G.C., $\mathscr{T}_{\mathrm{ab}}$.

\section{B. Expression des réponses et paramètres de l'interaction}

On cherche à exprimer les réponses des populations $\mathrm{P}_{\mathrm{a}}$ et $\mathrm{P}_{\mathrm{ab}}$ aux différents systèmes de test préconisés en fonction des paramètres de l'interaction. Pour prédire ces réponses, on utilise la méthode exposée par GALLAIs (1978) de régression linéaire entre la valeur phénotypique $T_{i}$ d'un candidat selon le système de test $\mathscr{T}_{i}$ (critère de sélection) et la valeur génotypique $M_{j}$ de sa descendance selon le système de reproduction $\mu_{j}$, en supposant linéaire la liaison entre $T_{i}$ et $\mathbf{M}_{j}$. 


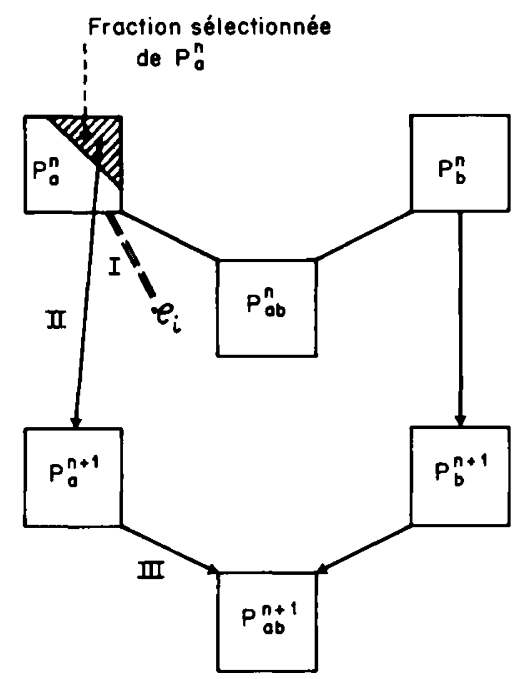

I Phase de test des candidats de $\mathrm{P}_{\mathrm{a}}^{\mathrm{n}}$ selon le système de test $\mathscr{T}_{\mathrm{i}}$.

II Phase de reproduction intra-population des individus sélectionnés de $\mathrm{P}_{\mathrm{a}}$.

III Phase de reproduction de $\mathrm{P}_{\mathrm{a}}^{\mathrm{n}+1}$ en croisement avec $\mathrm{P}_{\mathrm{b}}$ pour former $\mathrm{P}_{\mathrm{ab}}^{\mathrm{n}+1}$.

FIG. 1

Schéma d'un cycle (de rang $n$ ) de sélection de la population $P$ et de production de la population croisée $P_{a b}$ (d'après GALlaIs, 1978).

Diagram of one cycle $(n)$ of selection of population $P_{a}$ and of production of the crossbred population $P_{a b}$ (after GALlats, 1978).

Le progrès génétique $\Delta$, transmis par le système de reproduction $\mu_{\mathrm{j}}$ sachant qu'on a pratiqué le système de test $\mathscr{T}_{\mathrm{i}}$ s'écrit alors :

$$
\Delta\left(M_{j}, \mathscr{T}_{\mathrm{i}}\right)=\operatorname{cov}\left(\mathrm{M}_{\mathrm{j}}, \mathrm{T}_{\mathrm{i}}\right) \cdot \Delta \mathrm{T}_{\mathrm{i}} / \mathrm{v}\left(\mathrm{T}_{\mathrm{i}}\right)=\mathrm{k}_{\mathrm{i}} \operatorname{cov}\left(\mathrm{M}_{\mathrm{j}}, \mathrm{T}_{\mathrm{i}}\right)
$$

où $\mathrm{k}_{\mathrm{i}}=\Delta \mathrm{T}_{\mathrm{i}} / \mathrm{v}\left(\mathrm{T}_{\mathrm{i}}\right)$ et $\Delta \mathrm{T}_{\mathrm{i}}$ représente la différentielle de sélection dans le système de test $\mathscr{T}_{\mathrm{i}}$. Les réponses $\Delta \mathrm{P}_{\mathrm{a}}$ et $\Delta \mathrm{P}_{\mathrm{ab}}$ des populations $\mathrm{P}_{\mathrm{a}}$ et $\mathrm{P}_{\mathrm{ab}}$ s'obtiennent, quel que soit le système de test $\mathscr{T}_{\mathrm{i}}$, en prenant respectivement $\mu_{\mathrm{j}}=\mu_{\mathrm{aa}}$ et $\mu_{\mathrm{j}}=\mu_{\mathrm{ab}}$. Le tableau 2 donne les expressions de $T_{i}$, de $M_{j}$ et de $\operatorname{cov}\left(M_{j}, T_{i}\right)$ pour les 3 systèmes de test et les 2 systèmes de reproduction envisagés.

Expression de $T_{i}$ : En sélection massale, $\mathrm{T}_{\mathrm{a}}$ est la somme de la valeur génotypique du candidat $\left(G_{a a}\right)$ et d'une variable résiduelle $\left(Z_{a}\right)$ représentant l'effet du milieu. Avec des tests sur A.G.C., $T_{a a}$ (resp. $T_{a b}$ ), valeur phénotypique moyenne de la descendance du candidat intra-population (resp. en croisement) s'exprime en fonction de son A.G.C., $\Gamma_{\mathrm{aa}}\left(\right.$ resp. $\Gamma_{\mathrm{ab}}$ ) et d'un résidu $\mathrm{Z}_{\mathrm{aa}}$ (resp. $\mathrm{Z}_{\mathrm{ab}}$ ) non corrélé à $\Gamma$, incluant l'effet du milieu et l'erreur d'échantillonnage liée au choix des partenaires.

Expression de $M_{j}$ : La valeur $\mathrm{M}_{\mathrm{aa}}$ de la descendance d'un individu de $\mathrm{P}_{\mathrm{a}}$ selon $M_{\mathrm{aa}}$ est, par définition, son A.G.C. intra-population $\left(\Gamma_{\mathrm{aa}}\right)$. La valeur $\mathrm{M}_{\mathrm{ab}}$ de sa descendance selon $\mu_{\mathrm{ab}}$ s'exprime en fonction des composantes transmissibles de sa valeur génotypique, 


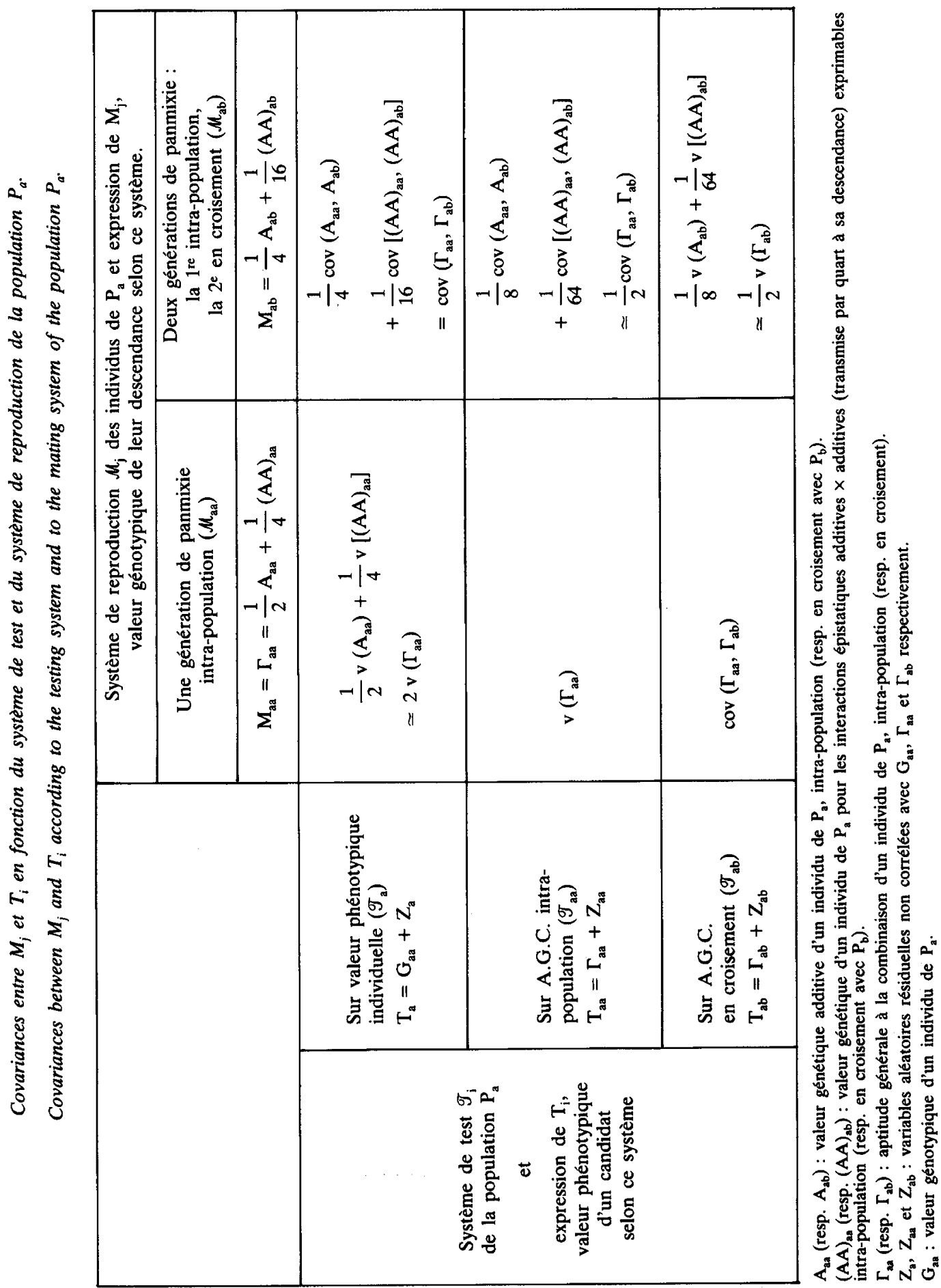


exprimées en croisement (BRUN, 1982) : ces composantes sont sa valeur génétique additive pour sa performance en croisement $\left(A_{a b}\right)$ et sa valeur pour les interactions épistatiques entre effets additifs. En se limitant aux interactions épistatiques d'ordre 2, $(\mathrm{AA})_{\mathrm{ab}^{\prime}}$ et en l'absence de linkage, $\mathrm{M}_{\mathrm{ab}}$ s'écrit $\frac{1}{4} \mathrm{~A}_{\mathrm{ab}}+\frac{1}{16}(\mathrm{AA})_{\mathrm{ab}}$. Les coefficients $\frac{1}{4}$ et $\frac{1}{16}$ traduisent le fait que la valeur additive et la valeur d'interaction additive $\times$ additive se transmettent respectivement par moitié et par quart à chaque génération de panmixie.

Dans certains cas, cov $\left(\mathrm{M}_{\mathrm{j}}, \mathrm{T}_{\mathrm{i}}\right)$ et donc la réponse à la sélection s'expriment directement en fonction de certains paramètres de l'interaction $v\left(\Gamma_{\mathrm{aa}}\right), \mathrm{v}\left(\Gamma_{\mathrm{ab}}\right)$ ou $\operatorname{cov}\left(\Gamma_{\mathrm{aa}}, \Gamma_{\mathrm{ab}}\right)$. Cela se produit notamment lorsque $\mathscr{T}_{\mathrm{i}}$ est un test sur aptitude générale à la combinaison et que $\mu_{\mathrm{j}}$ consiste en une seule génération de panmixie. Dans les autres cas, des approximations sur les variances et covariances d'interaction additive $\times$ additive permettent d'exprimer les réponses en fonction des paramètres de l'interaction.

\section{Conséquences pour l'estimation des paramètres de l'interaction}

L'observation des réponses et la connaissance des paramètres $\mathbf{k}_{\mathrm{i}}$, caractéristiques des systèmes de test, permettent d'estimer tous les paramètres de l'interaction.

En l'absence d'information sur les paramètres $k_{i}$, la pratique simultanée de 2 systèmes de tests (en pur et en croisement) et la simple mesure des réponses en pur et en croisement permettent d'estimer les 2 composantes de l'interaction : r, la corrélation entre A.G.C. en pur et en croisement et $\gamma$ le rapport des variances d'A.G.C. en pur et en croisement. Ainsi, par exemple, dans le cas de tests sur A.G.C. :

$$
\begin{gathered}
\mathrm{r}^{2}\left(\Gamma_{\mathrm{aa}}, \Gamma_{\mathrm{ab}}\right)=\frac{\Delta \mathrm{P}_{\mathrm{a}}\left(\mathscr{T}_{\mathrm{ab}}\right) \Delta \mathrm{P}_{\mathrm{ab}}\left(\mathscr{T}_{\mathrm{aa}}\right)}{\Delta \mathrm{P}_{\mathrm{ab}}\left(\mathscr{T}_{\mathrm{ab}}\right) \Delta \mathrm{P}_{\mathrm{a}}\left(\mathscr{T}_{\mathrm{aa}}\right)} \\
\gamma_{\mathrm{aa}, \mathrm{ab}}=\frac{\mathrm{v}\left(\Gamma_{\mathrm{aa}}\right)}{\mathrm{v}\left(\Gamma_{\mathrm{ab}}\right)}=4 \cdot \frac{\Delta \mathrm{P}_{\mathrm{a}}\left(\mathscr{T}_{\mathrm{ab}}\right) \Delta \mathrm{P}_{\mathrm{a}}\left(\mathscr{T}_{\mathrm{aa}}\right)}{\Delta \mathrm{P}_{\mathrm{ab}}\left(\mathscr{T}_{\mathrm{ab}}\right) \Delta \mathrm{P}_{\mathrm{ab}}\left(\mathscr{T}_{\mathrm{aa}}\right)}
\end{gathered}
$$

\section{Possibilités et limites de construction d'un test de l'interaction à partir des résultats expérimentaux disponibles dans la littérature}

Les expériences de sélection à 2 populations susceptibles d'une interprétation en termes d'interaction géniteur $\times$ population partenaire sont :

- les expériences de sélection intra-population (S.I.P.) où 2 populations sont sélectionnées de façon classique sur des tests intra-population et où on évalue périodiquement, parfois à chaque génération, leurs croisements $F_{1}$ réciproques ;

- les expériences de sélection basées sur des tests en croisement qui sont de 2 types : la sélection récurrente à testeur constant et la sélection récurrente et réciproque (S.R.R.) où les 2 populations en présence sont sélectionnées et se servent de testeurs mutuels pour le test des candidats sur descendance croisée (COMSTOCK et al., 1949). Chez les animaux, la S.R.R. est de loin la plus souvent pratiquée. 
Certains expérimentateurs réalisent parallèlement les 2 types de méthodes de sélection qu'ils mettent en comparaison après avoir dupliqué les populations de base.

Un problème posé par l'interprétation de ces expériences réside dans le fait que les populations en présence sont toutes 2 sélectionnées, contrairement au modèle d'expérience proposé. Le tableau 3 donne l'expression des réponses des populations parentales $\left(P_{a}\right.$ et $P_{b}$ ) et de la population croisée $P_{a b}$ (en supposant l'absence de différences entre les croisements $F_{1}$ réciproques) pour les 2 méthodes de sélection envisagées : la sélection intra-population et la sélection récurrente et réciproque. Il se déduit du tableau 2 en supposant l'additivité des contributions de $\mathrm{P}_{\mathrm{a}}$ et $\mathrm{P}_{\mathrm{b}}$ au progrès de $\mathrm{P}_{\mathrm{ab}}$. Cette additivité est une conséquence de la linéarité des liaisons entre le critère de sélection $\mathrm{T}$ d'un géniteur et la valeur génotypique de sa descendance en croisement et de la non-corrélation entre les tests $T_{1}$ et $T_{2}$ faits pour la sélection dans $P_{a}$ et $P_{b}$, respectivement. Les hypothèses de linéarité s'expriment par les relations :

$$
\begin{aligned}
& \Delta \mathrm{P}_{\mathrm{ab}}\left(\Delta \mathrm{T}_{1}\right)=\beta_{1} \Delta \mathrm{T}_{1} \\
& \Delta \mathrm{P}_{\mathrm{ab}}\left(\Delta \mathrm{T}_{2}\right)=\beta_{2} \Delta \mathrm{T}_{2} \\
& \Delta \mathrm{P}_{\mathrm{ab}}\left(\Delta \mathrm{T}_{1}, \Delta \mathrm{T}_{2}\right)=\lambda_{1} \Delta \mathrm{T}_{1}+\lambda_{2} \Delta \mathrm{T}_{2}
\end{aligned}
$$

Si $T_{1}$ et $T_{2}$ sont des tests intra-population, ils s'appuient sur des observations d'animaux non apparentés et sont donc non corrélés. Alors $\lambda_{1}=\beta_{1}$ et $\lambda_{2}=\beta_{2}$ et l'hypothèse d'additivité est vérifiée.

$\mathrm{Si} \mathrm{T}_{1}$ et $\mathrm{T}_{2}$ sont des tests sur aptitude générale à la combinaison en croisement, leur non-corrélation est une propriété des aptitudes générales à la combinaison définies dans $\mathrm{P}_{\mathrm{ab}}($ BRUN, 1982).

Les réponses du tableau 3 font intervenir 6 paramètres de l'interaction (les 3 paramètres de l'interaction des géniteurs de $P_{a}$ en croisement avec $P_{a}$ ou $P_{b}$ et les 3 paramètres de l'interaction "réciproque ») et les paramètres des systèmes de tests $\left(\mathrm{k}_{\mathrm{aa}}\right.$ et $\mathrm{k}_{\mathrm{bb}}$ pour les tests intra-population et $\mathrm{k}_{\mathrm{ab}}$ et $\mathrm{k}_{\mathrm{ba}}$ pour les tests en croisement).

\section{TABleaU 3}

Réponses des populations parentales et de leur croisement à la sélection intra-population et à la sélection récurrente réciproque

en fonction des paramètres des interactions géniteur $\times$ population partenaire générées par les populations $P_{a}$ et $P_{b}$.

Purebred and crossbred responses to intra-population and reciprocal recurrent selection

\begin{tabular}{|c|c|c|c|}
\hline & $\begin{array}{l}\text { Réponse de } P_{a} \\
\Delta \mathrm{P}_{\mathrm{a}}\end{array}$ & $\begin{array}{l}\text { Réponse de la population croisée } \\
\qquad \Delta \mathrm{P}_{\mathrm{ab}}\end{array}$ & $\begin{array}{c}\text { Réponse de } P_{b} \\
\Delta \Delta \mathrm{P}_{\mathrm{b}}\end{array}$ \\
\hline $\begin{array}{l}\text { Sélection } \\
\text { intra- } \\
\text { population }\end{array}$ & $\mathrm{k}_{\mathrm{aa}} \cdot \mathrm{v}\left(\Gamma_{\mathrm{aa}}\right)$ & $\frac{1}{2}\left[k_{a a} \cdot \operatorname{cov}\left(\Gamma_{a a}, \Gamma_{a b}\right)+k_{b b} \operatorname{cov}\left(\Gamma_{b b}, \Gamma_{b a}\right)\right]$ & $\mathrm{k}_{\mathrm{bb}} \cdot \mathrm{v}\left(\Gamma_{\mathrm{bb}}\right)$ \\
\hline $\begin{array}{l}\text { Sélection } \\
\text { récurrente } \\
\text { et réciproque }\end{array}$ & $\mathbf{k}_{\mathrm{ab}} \cdot \operatorname{cov}\left(\Gamma_{\mathrm{aa}}, \Gamma_{\mathrm{ab}}\right)$ & $\frac{1}{2}\left[k_{a b} \cdot v\left(\Gamma_{a b}\right)+k_{b a} \cdot v\left(\Gamma_{b a}\right)\right]$ & $\mathrm{k}_{\mathrm{ba}} \cdot \operatorname{cov}\left(\Gamma_{\mathrm{bb}}, \Gamma_{\mathrm{ba}}\right)$ \\
\hline
\end{tabular}
(applied to populations $P_{a}$ and $P_{b}$ ) as functions of the parameters of the "breeding animal $\times$ population of mates interactions " between $P_{a}$ and $P_{b}$. 
Même dans la situation optimale où les 6 réponses figurant au tableau 3 sont observées et où les paramètres $\mathrm{k}$ sont connus, il n'est pas possible d'estimer tous les paramètres de l'interaction, car il n'y a pas indépendance linéaire entre les 6 expressions théoriques des réponses du tableau 3.

En pratique, les paramètres $\mathrm{k}$ ne sont pas toujours publiés. Que peut-on estimer dans ces conditions?

Dans le cas de la sélection intra-population, la réponse de la population $\mathrm{P}_{\mathrm{ab}}$ peut s'exprimer en fonction des réponses de $\mathrm{P}_{\mathrm{a}}$ et $\mathrm{P}_{\mathrm{b}}$ et des coefficients de régression de l'A.G.C. en croisement sur l'A.G.C. intra-population :

$$
\left.\Delta \mathrm{P}_{\mathrm{ab}}=\frac{1}{2} \mathrm{~b}\left(\Gamma_{\mathrm{ab}} / \Gamma_{\mathrm{aa}}\right) \cdot \Delta \mathrm{P}_{\mathrm{a}}+\frac{1}{2} \mathrm{~b}\left(\Gamma_{\mathrm{ba}} / \Gamma_{\mathrm{bb}}\right) \Delta \mathrm{P}_{\mathrm{b}}\right)
$$

Dans le cas de la sélection récurrente et réciproque, on a ;

$$
\Delta \mathrm{P}_{\mathrm{ab}}=\frac{1}{2}\left[1 / \mathrm{b}\left(\Gamma_{\mathrm{aa}} / \Gamma_{\mathrm{ab}}\right)\right] \Delta \mathrm{P}_{\mathrm{a}}+\frac{1}{2}\left[1 / \mathrm{b}\left(\Gamma_{\mathrm{bb}} / \Gamma_{\mathrm{ba}}\right)\right] \Delta \mathrm{P}_{\mathrm{b}}
$$

Cas $n^{\circ} 1$ : si l'on dispose dans une expérience sur $\mathrm{g}$ générations, de séries de valeurs conjointes $\left(\Delta \mathrm{P}_{\mathrm{ab}}^{\mathrm{n}} \Delta \mathrm{P}_{\mathrm{a}}^{\mathrm{n}}, \Delta \mathrm{P}_{\mathrm{b}}\right)$ où $\mathrm{n}$ est l'indice de la génération, on peut pratiquer une analyse de régression de $\Delta \mathrm{P}_{\mathrm{ab}}$ sur les variables explicatives $\Delta \mathrm{P}_{\mathrm{a}}$ et $\Delta \mathrm{P}_{\mathrm{b}}$. Les coefficients de cette régression estimeront les coefficients de $\Delta \mathrm{P}_{\mathrm{a}}$ et $\Delta \mathrm{P}_{\mathrm{b}}$ dans les relations (1) et (2).

$\mathrm{Si}$ les 2 expériences ont été faites, on aura, pour chaque interaction, le coefficient de corrélation $r$ entre A.G.C. en pur et en croisement et le rapport $\gamma$ des variances d'A.G.C. en pur et en croisement. Pour l'interaction à partir des géniteurs de $P_{a}$ par exemple, on a :

$$
\begin{aligned}
& \mathrm{r}^{2}\left(\Gamma_{\mathrm{aa}}, \Gamma_{\mathrm{ab}}\right)=\mathrm{b}\left(\Gamma_{\mathrm{ab}} / \Gamma_{\mathrm{aa}}\right) \cdot \mathrm{b}\left(\Gamma_{\mathrm{aa}} / \Gamma_{\mathrm{ab}}\right) \\
& \gamma_{\mathrm{aa}, \mathrm{ab}}=\mathrm{v}\left(\Gamma_{\mathrm{aa}}\right) / \mathrm{v}\left(\Gamma_{\mathrm{ab}}\right)=\mathrm{b}\left(\Gamma_{\mathrm{aa}} / \Gamma_{\mathrm{ab}}\right) / \mathrm{b}\left(\Gamma_{\mathrm{ab}} / \Gamma_{\mathrm{aa}}\right)
\end{aligned}
$$

Cas no 2 : Si l'on ne connaît pas les réponses par génération, on ne pourra plus séparer les contributions de $\mathrm{P}_{\mathrm{a}}$ et de $\mathrm{P}_{\mathrm{b}}$ au progrès de $\mathrm{P}_{\mathrm{ab}}$ et donc les 2 interactions réciproques. On fera l'hypothèse que leurs 2 composantes sont égales :

$$
\begin{gathered}
r\left(\Gamma_{\mathrm{aa}}, \Gamma_{\mathrm{ab}}\right)=\mathrm{r}\left(\Gamma_{\mathrm{bb}}, \Gamma_{\mathrm{ba}}\right)=\mathrm{r}_{\mathrm{pc}} \\
\text { et } \mathrm{v}\left(\Gamma_{\mathrm{aa}}\right) / \mathrm{v}\left(\Gamma_{\mathrm{ab}}\right)=\mathrm{v}\left(\Gamma_{\mathrm{bb}}\right) / \mathrm{v}\left(\Gamma_{\mathrm{ba}}\right)=\gamma_{\mathrm{pc}}
\end{gathered}
$$

Ces 2 relations sont équivalentes à l'unicité du coefficient $b_{\mathrm{p} / \mathrm{c}}$ de régression de l'A.G.C. en pur sur l'A.G.C. en croisement (et du coefficient $b_{c / p}$ de la régression inverse) quelle que soit la population d'origine des géniteurs. réalisés

En se reportant aux relations (1) et (2), on peut estimer les coefficients de régression $\mathrm{b}_{c / p}$, en sélection intra-population, par $\frac{\Delta \mathrm{P}_{\mathrm{ab}}}{\left(\Delta \mathrm{P}_{\mathrm{a}}+\Delta \mathrm{P}_{\mathrm{b}}\right) / 2}$

et $b_{p / c}$, en sélection récurrente et réciproque, par $\frac{\left(\Delta P_{a}+\Delta P_{b}\right) / 2}{\Delta P_{a b}}$ 
Dans les 2 cas, ce coefficient de régression s'obtient simplement, en faisant le rapport de la réponse corrélée moyenne à la réponse directe moyenne.

Lorsque les 2 méthodes de sélection sont réalisées simultanément, on peut estimer la corrélation génétique pur-croisement et le rapport des variances en pur et en croisement par :

$r_{p c}^{2}=b_{c / p} \cdot b_{p / c}$ et $\gamma_{p c}=b_{p / c} / b_{c / p}$

Cette approche de l'interaction par la comparaison des réponses en pur et en croisement, rencontre une autre limite importante, liée à l'existence de consanguinité dans les populations parentales: une dépression de consanguinité peut affecter les souches pures sélectionnées, restaurée par le croisement ; on risque alors d'interpréter comme des interactions géniteur $\times$ population partenaire une divergence croissante entre les moyennes des souches pures et de leurs croisements, dont une partie est en fait liée à l'hétérosis croissant entre les 2 souches en présence. Si l'on dispose de 2 souches témoins et de leurs croisements, on peut éliminer l'effet de la consanguinité en exprimant chaque réponse en écart à son témoin respectif et en admettant d'une part que la dépression de consanguinité est la même dans les souches témoins et sélectionnées et d'autre part, que les effets de la sélection et de la consanguinité n'interagissent pas.

\section{Discussion}

Les paramètres de l'interaction géniteur $\times$ population partenaire que l'on peut calculer à partir des expériences de sélection à 2 populations sont très imprécis : aux sources d'imprécision, étudiées par HiLL (1972), sur les paramètres génétiques réalisés (variance d'échantillonnage des lignées et variance de dérive) peuvent s'ajouter des biais liés au non respect des hypothèses que l'on a dû faire : celle de l'identité des 2 interactions « réciproques » et celle d'absence d'interaction entre les effets de la sélection et de la consanguinité sur la moyenne des populations. Par ailleurs, le coefficient de régression pur-croisement ne constitue pas une mesure de l'interaction, à la différence du coefficient de corrélation. Tout au plus, constitue-t-il un indicateur de la présence d'interaction, lorsqu'il s'écarte de 1 . En outre, la validité des calculs qui reposent sur une double expérience de sélection suppose l'identité des paramètres de l'interaction lorsqư'elle s'exprime en sélection intra-population et en sélection récurrente réciproque, donc la stabilité des paramètres au cours des expériences. Or, la génétique quantitative prévoit une diminution de la corrélation génétique pur-croisement dans le cas particulier de la sélection récurrente réciproque (PIRCHNER \& MERGL, 1977), notamment en cas de superdominance, se traduisant par une non-linéarité de la réponse intra-population. Dans de tels cas, le test de l'interaction devra être pratiqué en fractionnant l'expérience en intervalles sur lesquels on pourra considérer les réponses comme linéaires.

On a utilisé une méthode peu classique (GALLAIS, 1978) pour prédire le progrès génétique des populations pures et croisées, tenant compte de l'ensemble des effets génétiques transmissibles, et notamment la part des interactions épistatiques additives $x$ additive transmise par les gamètes. L'approche classique pour estimer le progrès génétique se base sur l'estimation des valeurs génétiques additives au sens strict des candidats, pour leur performance en pur $\left(A_{p}\right)$ et en croisement $\left(A_{c}\right)$ (STANDAL, 1968). Avec cette dernière approche, les réponses des populations étudiées auraient eu des 
expressions similaires, avec les paramètres $v\left(A_{p}\right), v\left(A_{c}\right), \operatorname{cov}\left(A_{p}, A_{c}\right)$ au lieu des paramètres $v\left(\Gamma_{p}\right), v\left(\Gamma_{c}\right), \operatorname{cov}\left(\Gamma_{p}, \Gamma_{c}\right)$. Cette méthode n'aurait donc pas permis d'exprimer les réponses en fonction des paramètres de l'interaction.

Les formules prédictives que nous avons utilisées, permettent d'analyser les implications en amélioration génétique des interactions géniteur $\times$ population partenaire : lorsque l'objectif de la sélection est d'améliorer le croisement entre 2 populations complémentaires, on pratique fréquemment une sélection intra-population - c'est une sélection indirecte - en espérant que les gains génétiques ainsi obtenus dans les 2 populations se cumuleront au niveau du croisement. Or, il existe un seuil d'interaction qui peut rendre préférable une sélection directe, basée sur des tests en croisement, comme la sélection récurrente et réciproque. En supposant, pour une méthode de sélection donnée, l'égalité des paramètres (génétiques ou non) relatifs aux 2 populations en présence, le rapport $\mathrm{R}$ de la réponse directe (par génération) à la réponse indirecte s'écrit :

$$
\mathrm{R}=\theta \frac{\mathrm{v}\left(\Gamma_{\mathrm{c}}\right)}{\operatorname{cov}\left(\Gamma_{\mathrm{p}^{\prime}}, \Gamma_{\mathrm{c}}\right)}=\theta /\left(\mathrm{r}_{\mathrm{pc}} \cdot \sqrt{\gamma_{\mathrm{pc}}}\right)
$$

où $\theta$ est une fonction des intervalles de générations, différentielles de sélection et écart-type du critère de sélection dans les 2 méthodes. Cette relation met en évidence la contribution des 2 composantes de l'interaction au choix d'un système de sélection en vue du croisement.

\section{Conclusion}

Cette étude montre comment planifier une expérience de sélection utilisant 2 populations pour détecter les interactions géniteur $\times$ population partenaire. Par ailleurs, elle fournit un indicateur de l'interaction que l'on peut facilement calculer à partir d'expériences déjà réalisées de sélection intra-population et de sélection récurrente et réciproque : en appelant « directe » la réponse obtenue dans le milieu génétique de la sélection et "corrélée " celle obtenue dans l'autre milieu, cet indicateur est le rapport de la réponse corrélée moyenne à la réponse directe moyenne des 2 populations. Il constitue le coefficient de régression réalisé de l'aptitude générale à la combinaison en croisement sur l'aptitude générale à la combinaison intra-population ou inversement.

Cette approche de l'interaction sera utilisée lors d'une analyse bibliographique de résultats expérimentaux et confrontée à une autre approche, reposant sur l'estimation statistique des paramètres de l'interaction.

Reçu le 6 mai 1983.

Accepté le 21 février 1984.

\section{Remerciements}

L'auteur remercie tout particulièrement C. Chevalet et R. Rouvier ainsi que les lecteurs mandatés par la revue pour leurs critiques et suggestions lors de la lecture du manuscrit. 


\section{Références bibliographiques}

BRUN J.M., 1982. Interactions géniteur $\times$ population des partenaires. 1: Définition d'indicateurs. Ann. Génét. Sél. Anim., 14, 463-479.

Comstock R.E., Robinson M.F., HARVEY P.H., 1949. A breeding procedure designed to make maximum use of both general and specific combining ability. J. Am. Soc. Agron., 41, 360-367.

Gallais A., 1978. Amélioration des populations, méthodes de sélection et création de variétés. III. Bases théoriques pour l'étude de la sélection récurrente réciproque. Ann. Amélior. Plant., 28, 637-666.

HILL W.G., 1972. Estimation of realised heritabilities from selection experiments. Biometrics, 28, 747.780.

PirChner F., Mergl R., 1977. Overdominance as cause for heterosis in poultry. Z. Tierz. Züchtgsbiol., 94, 151-158.

STANDal N., 1968. Studies on breeding and selection schemes in pigs. I. Selection on performance of purebred versus crossbred progeny. Acta. Agric. Scand., 18, 222-232. 